\title{
Age effect on in vitro fertilization pregnancy mediated by anti-Mullerian hormone (AMH) and modified by follicle stimulating hormone (FSH)
}

\author{
Han-Chih Hsieh ${ }^{1}$, Jia-Ying Su', Shunping Wang ${ }^{2}$ and Yen-Tsung Huang ${ }^{1 *}$
}

\begin{abstract}
Background: Both follicle stimulating hormone (FSH) and anti-mullerian hormone (AMH) are widely used to assess the ovarian reserve in women for in vitro fertilization (IVF). However, studies also showed that both AMH and FSH are significantly associated with age: as age increases, AMH decreases and FSH increases. This study aims to investigate the mechanism of age effect on IVF live birth rate, particularly through mediation and interaction by AMH and FSH.

Methods: We conducted a retrospective cohort study of 13970 IVF cycles collected by elVF from 2010 to 2016. A series of logistic mixed models were used to estimate the association of live birth and AMH (or FSH). The mediation effects and proportion mediated, were quantified by our previously proposed mediation analyses. We further investigated the FSH-modified mediation effects on live birth rate through AMH, accounting for the nonlinear age effect.

Results: Our analyses showed that age effect on live birth was mediated more by AMH than by FSH (18 vs. $6 \%$ ). The mediation effect through AMH can be further modified by FSH level regardless of women's age.

Conclusions: In summary, mediation model provides a new perspective elucidating the mechanism of IVF successful rate by age. The majority of the age effect on live birth rate remained unexplained by AMH and FSH, suggesting its importance and independent role in IVF.
\end{abstract}

Keywords: Anti-Mullerian hormone, Follicle stimulating hormone, In vitro fertilization, Mediation analysis

\section{Background}

For female infertility patients, follicle stimulating hormone (FSH) and anti-müllerian hormone (AMH) represent the two most frequently utilized ovarian reserve tests that serve as important markers reflecting successful in vitro fertilization (IVF) outcomes. FSH is a member of the glycoprotein hormone family, produced by the gonadotropic cells of the anterior pituitary gland [1]; AMH is a dimeric glycoprotein hormone exclusively

\footnotetext{
*Correspondence: ythuang@stat.sinica.edu.tw

'Institute of Statistical Science, Academia Sinica, 128 Academia Road, 11529

Taipei, Taiwan

Full list of author information is available at the end of the article
}

produced by granulosa cells [2-4]. Previous work suggested that low AMH confers to a lower likelihood of live birth, while low FSH confers to a higher likelihood of live birth [5-7]. Yet, it is suggested that AMH $>0.8-1.0$ $\mathrm{ng} / \mathrm{ml}$ is indicative of normal ovarian reserve, while FSH $>10 \mathrm{mIU} / \mathrm{ml}$ is indicative of diminished ovarian reserve [6]. In addition to the dose-response relationship between $\mathrm{AMH}$ (or FSH) and the probability of live birth, both markers are also found to be significantly associated with age: as age increases, AMH decreases and FSH increases [8-10]. Furthermore, numerous studies have identified that as women's age increases, the success live birth rate of IVF decreases [11-13]. 
Based on the associations mentioned above, this study aims to further investigate the mechanism of age effect on IVF live birth rate, particularly through mediation by $\mathrm{AMH}$ and FSH. Mediation serves as a mechanism linking a risk factor (e.g., age) and an outcome (e.g., live birth rate). Mediation analysis was first proposed in psychology literature where mediation effect was identified by a product of two associations: one between the risk factor and the mediator and the other between the mediator and the outcome conditional on the risk factor $[14,15]$. For the past two decades, mediation analysis has been extensively developed under the framework of causal inference $[16,17]$. Using the notions of counterfactual outcome [18], causal definitions and their identifiability assumptions have been carefully studied [19, 20]. In addition to providing rigorous theoretical foundation, causal mediation analysis enables us to conduct mediation analyses for the setting where the mediator and the outcome are noncontinuous with possible exposure-by-mediator nonlinear interactions [21-23]. We framed our scientific question by a mediation model where we had a dichotomous AMH or FSH (high vs. low) and a dichotomous live birth outcome (yes vs. no), and the effects of interest were the direct effect (DE) of age on the risk of live birth and the indirect effect (IE) mediated through AMH or FSH.

The main purposes of this study were therefore to quantify the mediation effects of age on live birth through $\mathrm{AMH}$ or $\mathrm{FSH}$, incorporating the interactions between age and both intermediary factors. Additionally, we examined whether the age effect on live birth mediated through $\mathrm{AMH}$ is further mediated by FSH level.

\section{Methods}

\section{Patient selection}

A retrospective cohort analysis was conducted using the electronic medical record database eIVF, designed by PracticeHwy.com (Dallas, Texas). The dataset we obtained consisted of 144,044 cycles extracted from 60 different IVF centers in the United States from 2000 to 2016. Information recorded in the eIVF database for each cycle included a distinctive numeric patient identifier, patient's age at cycle start, the date of the oocyte retrieval, cycle status, cycle outcome, AMH, FSH, estradiol, along with other 80 variables. Our work involved the de-identified eIVF dataset and was determined to be exempt by Institutional
Review Board of Women and Infants Hospital of Rhode Island.

Since the assessment of AMH had not been widely adopted in clinical use until the year of 2010, cycles before 2010 were excluded resulting from the missing AMH values. Cycles with missing or incomplete information were all excluded, as well as those were outliers in certain variables, such as age, cycle number, total number of oocytes retrieved, number of 2PNs, number of follicles, the value of E2, AMH and FSH. Centers that provided with less than 10 cycles were considered unreliable, thus were removed. Therefore, the final dataset contained 13790 autologous IVF cycles, with known AMH, FSH values and confirmed information about live birth or not. Details about the inclusion and exclusion criteria of the study were documented as a flowchart in Fig. 1 in the previous paper [24] and in our Supplementary Materials.

\section{Statistical methods}

To observe the marginal effects of age, AMH and FSH, a series of logistic mixed effect models were used to investigate the association of live birth and AMH (or FSH), adjusted for potential confounders: age, body mass index (BMI) and random effects of centers, as well as involving the interactions between age, AMH and FSH. Random effects of centers were included to account for the heterogeneity across numerous centers.

Figure 1 shows the two causal diagrams that depict the direct (blue) and indirect (red) effects in a mediation model. One with AMH, the other with FSH as the mediator; both with age as the exposure and live birth as the outcome. In order to fit the single-mediator model we developed based on the methods estimating mediation effect of both dichotomous mediator and outcome [23], we converted the original continuous $\mathrm{AMH}$ and $\mathrm{FSH}$ values into dichotomous values to reflect their clinical significance: AMH less than $1.0 \mathrm{ng} / \mathrm{ml}$ and FSH less than 10 $\mathrm{mIU} / \mathrm{ml}$ were considered as $0 \mathrm{~s}$; FSH greater than or equal to $10 \mathrm{mIU} / \mathrm{ml}$ and $\mathrm{AMH}$ values greater than or equal to $1.0 \mathrm{ng} / \mathrm{ml}$ were considered as $1 \mathrm{~s}$. We then calculated the risk ratios $(\mathrm{RR})$ and confidence intervals $(\mathrm{CI})$ of the direct effects (DE) and indirect effects (IE) through AMH and FSH with and without adjusting the interaction with age, by using the bootstrap method and the mediation models we proposed. We reported direct and indirect effects on
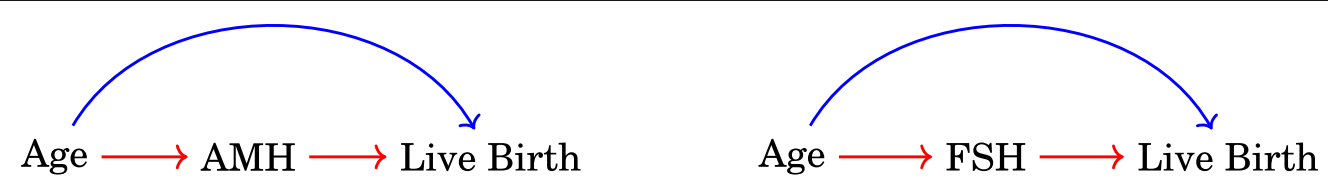

Fig. 1 Causal diagrams with age as the exposure, AMH or FSH as the mediator, and live birth as the outcome 
the scale of risk ratios (RR), comparing the live birth rate of women with age of 39 (the 3rd quantile) vs 32 (the 1st quantile).

Additional mediation analysis was conducted with cycles grouped based on the cut-off values we derived from using the quintiles of FSH (Q1=5.1, Q2=6.5, Q3=7.7, $\mathrm{Q} 4=9.7 \mathrm{mIU} / \mathrm{ml}$ ). Mediation models were applied to each groups by FSH level to identify the direct and indirect effects of age on live birth in relation to AMH. Furthermore, in order to take the nonlinear effect of age into consideration, cycles were also grouped based on patients' age: all ages were rounded to the nearest integer, while patients $<30$ years of age were considered as 30 , and patients $>40$ years of age were considered as 40 . By sorting the cycles based on their FSH and age, we then obtained a 5-by-11 matrix, each unit within the matrix contained cycles with FSH and age under certain cutoffs. The first group out of the 55, with the least FSH $(<5.1$ $\mathrm{mIU} / \mathrm{ml})$ and age $(<30$ years) value, was defined as the reference group. Mediation analysis was performed on the rest 54 groups, comparing to the reference, to determine the direct effects, indirect effects, and proportion mediated. The proportion mediated was defined as the proportion of indirect effect in the total effects; more specifically, $\log$ risk ratios of indirect effect divided by the sum of log risk ratios of direct effect and log risk ratios of indirect effect.

\section{Results}

The demographic characteristics and live birth rate among patients of age $30,35,37$, and 40 years old can be found in Table 1 . The average age (year \pm standard deviation (SD)) of the patient population is $35.0 \pm 4.7$. FSH significantly increased from $6.5 \mathrm{mIU} / \mathrm{ml}$ to $8.8 \mathrm{mIU} / \mathrm{ml}$ as age increased from $<30$ to $>40$ years old $(p<0.001)$, while AMH decreased significantly from $4.0 \mathrm{ng} / \mathrm{ml}$ to 1.2 $\mathrm{ng} / \mathrm{ml}(p<0.001)$. The live birth rate had a statistically significant decrease of $28.9 \%$ as the ages increased from $<30$ to $>40$ years old $(35.9 \%$ vs $7.0 \%, p<0.001)$.

As shown in Table 2, logistic regression models for live birth, AMH and FSH were performed using age, AMH,
FSH, adjusting the confounding factors and exposuremediator interactions. Simple regression models were performed to assess the marginal effects of age, AMH and FSH, showing that all three were notably associated with live birth $(p<0.001)$. As expected, the doseresponse relationship between AMH and live birth was positive, and the dose-response relationship between FSH and live birth was negative. To explore whether including the exposure (age)-mediator (AMH or FSH) interaction terms might improve the models, several more regressions were performed that supported significant age-by-AMH (or FSH) interactive effect on live birth $(p<0.05)$. We also performed regressions that included both $\mathrm{AMH}$ and FSH as well as their interaction terms with age. While the interaction between FSH and age was significantly associated with live birth, the interaction between AMH and age was not. Additional logistic regression models, shown in Table 3, were perform to estimate the marginal effects of age and FSH on AMH, adjusting the same confounding factors. Similarily, effects of age and AMH on FSH were also estimated. Both mediators turned out to be significantly associated with one another. $(p<0.001)$

A single-mediator model was used to assess the effects of both AMH and FSH on the probability of live birth. With the Q1 and Q3 of exposure we derived earlier, the results were observed in Table 4 that comparing patients who were 39 years old to those 32 years old, the effect mediated through AMH accounted 16\% (95\% CI: $14,18 \%)$ of the total effect by age, the effect mediated through FSH accounted for only 4\% (95\%CI: 4, 5\%). Additional mediation analysis conducted by involving the exposure-mediator interactions revealed similar results (18\% vs 6\%).

Table 5 demonstrates the direct effect (DE), indirect effect (IE), and proportion mediated obtained from applying our mediation model to cycles grouped based on the quintiles of FSH. Since AMH played a more important role in mediation as shown in Table 4, here we considered AMH as the mediator, and FSH as the effect modifier. Results showed that comparing patients with an average of 39 years old to those with an average of 32 years old,

Table 1 Demographic characteristics and live birth rate of all five different age groups

\begin{tabular}{|c|c|c|c|c|c|c|c|}
\hline \multirow{4}{*}{$\mathrm{N}$} & \multirow{2}{*}{ All } & \multicolumn{6}{|l|}{ Age } \\
\hline & & $<30$ & $30-35$ & $35-37$ & $37-40$ & $>40$ & \\
\hline & 13790 & 2202 & 4739 & 1913 & 2763 & 2173 & \\
\hline & Mean (SD) & Mean (SD) & Mean (SD) & Mean (SD) & Mean (SD) & Mean (SD) & $p$-values \\
\hline $\mathrm{BMI}\left(\mathrm{kg} / \mathrm{m}^{2}\right)$ & $25.9(6.0)$ & $26.0(6.2)$ & $25.7(6.0)$ & $26.1(5.9)$ & $26.1(5.9)$ & $26.1(5.8)$ & 0.012 \\
\hline Estradiol $(\mathrm{pg} / \mathrm{ml})$ & $2261(1485)$ & $2732(1673)$ & 2436 (1508) & 2277 (1483) & 1950 (1274) & $1751(1220)$ & $<0.001$ \\
\hline $\mathrm{FSH}(\mathrm{mlU} / \mathrm{ml})$ & $7.6(3.8)$ & $6.5(2.9)$ & $7.2(3.3)$ & $7.6(3.9)$ & $8.2(4.1)$ & $8.8(4.6)$ & $<0.001$ \\
\hline $\mathrm{AMH}(\mathrm{ng} / \mathrm{ml})$ & $2.4(2.7)$ & $4.0(3.4)$ & $2.9(2.9)$ & $2.1(2.3)$ & $1.5(1.7)$ & $1.2(1.3)$ & $<0.001$ \\
\hline Live Birth (\%) & 23.5 & 35.9 & 29.9 & 23.5 & 15.4 & 7.0 & $<0.001$ \\
\hline
\end{tabular}


Table 2 Odds ratio estimates on live birth as the outcome

\begin{tabular}{|c|c|c|c|c|c|c|}
\hline Live Birth & Model & Age & $\mathrm{AMH}$ & FSH & $\mathrm{AMH}^{*} \mathrm{Age}$ & $\mathrm{FSH}^{*}$ Age \\
\hline \multirow{3}{*}{ Marginal Effect } & M1 & $0.89(0.88,0.90)$ & - & - & - & - \\
\hline & M2 & - & $2.32(2.11,2.54)$ & - & - & - \\
\hline & M3 & - & - & $0.55(0.49,0.62)$ & - & - \\
\hline \multirow{4}{*}{ Individual Effect } & M4 & $0.90(0.89,0.91)$ & $1.68(1.52,1.86)$ & - & - & - \\
\hline & M5 & $0.89(0.88,0.90)$ & - & $0.69(0.61,0.78)$ & - & - \\
\hline & M6 & $0.88(0.87,0.90)$ & $0.71(0.33,1.55)$ & - & $1.03(1.00,1.05)$ & - \\
\hline & M7 & $0.89(0.89,0.90)$ & - & $2.63(0.97,7.10)$ & - & $0.96(0.94,0.99)$ \\
\hline \multirow{2}{*}{ Joint Effect } & M8 & $0.90(0.89,0.91)$ & $1.61(1.45,1.78)$ & $0.80(0.71,0.91)$ & - & - \\
\hline & M9 & $0.89(0.88,0.91)$ & $0.87(0.39,1.96)$ & $2.45(0.87,6.89)$ & $1.02(1.00,1.04)$ & $0.97(0.94,1.00)$ \\
\hline
\end{tabular}

M1-M3 are logistic regression models for live birth assessing the marginal effects of age, AMH and FSH adjusting bmi and random effect of centers.

M4-M7 are logistic regression models for live birth assessing the individual effects of AMH and FSH adjusting age, bmi, random effect of centers, and considering the interactions between age, $\mathrm{AMH}$ and $\mathrm{FSH}$.

M8-M9 are logistic regression models for live birth assessing the joint effects of AMH and FSH adjusting age, bmi, random effect of centers, and considering the interactions between age, $\mathrm{AMH}$ and FSH.

For M1-M9, their respective variances of the random effects estimates are $0.47,0.41,0.45,0.44,0.46,0.46,0.44,0.43,0.44$

the mediation effect through AMH accounted for $20 \%$ (95\%CI: 14,26\%) among patients with the least FSH value $(<5.1 \mathrm{mIU} / \mathrm{ml}, n=2720)$, which decreased monotonically to $7 \%$ (95\%CI: $2,11 \%)$ for patients with the highest FSH value ( $>9.7 \mathrm{mIU} / \mathrm{ml}, n=2766)$. The result suggested that AMH mediated the age effect on live birth (7\%-20\%), which was modified by FSH level.

An additional analysis was conducted in order to further explore the nonlinear effect of age. As shown in Fig. 2, where total effect (TE), direct effect (DE), indirect effect (IE) and proportion mediated are presented in heatmaps. The increase of direct effect among different age and FSH groups was mostly dominated by the increase of age (Fig. 2b); whereas the increase of indirect effect among different age and FSH groups was mostly dominated by the increase of FSH (Fig. 2c). However, all heatmaps indicated the trend that the total effect (TE), direct effect (DE), indirect effect (IE) increase as the FSH and age increase. Therefore, the heatmaps provided a comprehensive visualization of the path-specific effects between age, AMH and live birth, modifying age and FSH values. The analysis incorporating the nonlinear age effect further supported the result in Table 5 . The indirect effect of age increased mediated through AMH significantly decreased the live birth rate, and such mediation effects were notably modified by FSH level regardless of women's age.

\section{Discussion}

The principal finding of this study was that the age effect on live birth was more strongly mediated by AMH and less by FSH. By comparing patients who were 39 years old to those 32 years old, $16 \%$ of live birth resulted from a specific pathway: age affected AMH, which then in return affected live birth; FSH, on the other hand, accounted for only $4 \%$ of the age effect on live birth, indicating that FSH is a less significant mediator in the effect of age on live birth. However, aside from the statistically significant mediation effects through AMH and FSH, the direct effect of age still accounted for the majority of live births, which

Table 3 Odds ratio estimates on AMH and FSH as the outcomes

\begin{tabular}{|c|c|c|c|c|c|}
\hline & & Model & Age & $\mathrm{AMH}$ & $\mathrm{FSH}$ \\
\hline \multirow{3}{*}{$\mathrm{AMH}$} & \multirow{2}{*}{ Marginal Effect } & M1 & $0.84(0.84,0.85)$ & - & - \\
\hline & & M2 & - & - & $0.18(0.16,0.20)$ \\
\hline & Individual Effect & M3 & $0.85(0.85,0.86)$ & - & $0.21(0.19,0.23)$ \\
\hline \multirow{3}{*}{ FSH } & \multirow{2}{*}{ Marginal Effect } & M4 & $1.11(1.10,1.12)$ & - & - \\
\hline & & M5 & - & $0.18(0.16,0.20)$ & - \\
\hline & Individual Effect & M6 & $1.06(1.04,1.07)$ & $0.21(0.19,0.23)$ & - \\
\hline
\end{tabular}

M1-M2 are logistic regression models for AMH assessing the marginal effects of age and FSH, adjusting bmi and random effect of centers. $\mathrm{M} 3$ is a logistic regression model for AMH assessing the individual effect of FSH adjusting age, bmi, random effect of centers.

M4-M5 are logistic regression models for FSH assessing the marginal effects of age and AMH, adjusting bmi and random effect of centers. $\mathrm{M6}$ is a logistic regression model for FSH assessing the individual effect of AMH adjusting age, bmi, random effect of centers.

For M1-M6, their respective variances of the random effects estimates are $0.49,0.62,0.53,0.26,0.28,0.27$ 
Table 4 Risk ratio estimates of direct and indirect effects of age on live birth

\begin{tabular}{|c|c|c|c|c|c|}
\hline & Direct Effect & & Indirect Effect & & Proportion Mediated \\
\hline & Risk Ratio* & $p$-values & Risk Ratio* & $p$-values & Proportion \\
\hline $\mathrm{AMH}$ & $0.57(0.54,0.59)$ & $<0.001$ & $0.90(0.89,0.90)$ & $<0.001$ & $0.16(0.14,0.18)$ \\
\hline FSH & $0.53(0.50,0.56)$ & $<0.001$ & $0.97(0.96,0.97)$ & $<0.001$ & $0.05(0.04,0.06)$ \\
\hline $\mathrm{AMH}+\mathrm{int}$ & $0.57(0.54,0.60)$ & $<0.001$ & $0.88(0.87,0.90)$ & $<0.001$ & $0.18(0.16,0.21)$ \\
\hline $\mathrm{FSH}+\mathrm{int}$ & $0.53(0.50,0.56)$ & $<0.001$ & $0.96(0.95,0.97)$ & $<0.001$ & $0.06(0.05,0.08)$ \\
\hline
\end{tabular}

*Effects were estimated for the comparison of live birth rate between women with 39 years old and 32 years old.

suggests that age affected live birth through pathways other than through AMH or FSH.

The etiology of age affecting live birth rates through pathways other than AMH or FSH may be multi-factorial. Currently available diagnostic tests that are heavily influenced by women's age, such as estradiol, inhibin $\mathrm{B}$, the antral follicle count (AFC), the ovarian volume (OVVOL) and the ovarian blood flow, are also measures to predict the ovarian reserve and chance of live birth besides AMH and FSH [6]. Moreover, the reduction of live birth rates is not linked solely to the reduction of number of oocytes, but also to the reduction in the number of embryos and implantation rate, which studies have also found to be affected by age [11]. The biology underlying the association is potentially related to the impaired decidual and placental development and embryo interaction with the uterus [25]. In addition, medical disorders that are developed more commonly in older women, such as high blood pressure or diabetes, can lead to higher risk of fatal death, thus decrease the live birth rates [26].

Of note, since the interaction effects between $\mathrm{AMH}$ (or FSH) and age were statistically significant as shown in Table 2, the interaction terms were thus added in the mediation models to accommodate the relationships, yielding a more positive finding. The interaction effects indicate that even with the same levels of $\mathrm{AMH}$ or FSH, the resulting live births of every cycles under patients with different age may still vary. According to the estimates of the random effect of centers in both Tables 2 and 3, there exists variation for the effect of different centers across the United States on the live birth rate, and the mixed model effectively accounts for the variability of center effects. The age-varying effects may suggest that markers of ovarian aging or uterus aging other than AMH or FSH affecting the probability of live birth contribute to the effects, such as inhibin B, estradiol, antral follicle count(AFC) and uterine factors [27]. More studies are needed to provide additional evidence for the roles of these biomarkers determining the interaction effects between age and AMH (or FSH).

Through categorizing FSH and applying the mediation analyses, we were able to identify that the proportion mediated by AMH on live birth decreased as FSH value increased (Table 5). This finding suggests that the effects of AMH on live birth may vary depending on FSH level and that the increase of FSH would decrease the mediation effect of AMH. However, this approach, as well as the previous analysis, underlay the assumption of the linear relationship between age and live birth. The nonlinear effect between age and live birth has been well-documented by previous studies [28-30]. To account for this, the analysis presented in Fig. 2 was conducted using heatmaps, providing more sophisticated results on interpreting the mediation effects of AMH. Interestingly, although the direct effect (DE) of age, decreased the live birth rate as age increased; whereas the indirect effect (IE) of age through AMH decreased the live birth rate as FSH increased. The interaction between FSH and AMH presenting in the indirect effect (IE) of age on live birth rate through AMH (Fig. 2c), was supported by early studies where both markers were found regulating the development of ovary and follicular growth at different phases.

Table 5 Risk ratio estimates of direct and indirect effects of age on live birth with AMH as the mediator for different FSH groups

\begin{tabular}{llllll}
\hline FSH (N) & \multicolumn{2}{c}{ Direct Effect } & & \multicolumn{1}{c}{ Indirect Effect } & Proportion Mediated \\
\hline & Risk Ratio* & $p$-values & Risk Ratio* & $p$-values & Proportion \\
$<5.1(2720)$ & $0.63(0.55,0.69)$ & $<0.001$ & $0.89(0.87,0.91)$ & $<0.001$ & $0.20(0.14,0.26)$ \\
$5.1 \sim 6.5(2743)$ & $0.60(0.53,0.67)$ & $<0.001$ & $0.92(0.90,0.94)$ & $<0.001$ & $0.14(0.10,0.20)$ \\
$6.5 \sim 7.7(2699)$ & $0.65(0.58,0.72)$ & $<0.001$ & $0.93(0.91,0.95)$ & $<0.001$ & $0.14(0.09,0.20)$ \\
$7.7 \sim 9.7(2862)$ & $0.61(0.54,0.67)$ & $<0.001$ & $0.94(0.93,0.96)$ & $<0.001$ & $0.11(0.07,0.15)$ \\
$>9.7(2766)$ & $0.45(0.38,0.51)$ & $<0.001$ & $0.95(0.92,0.98)$ & 0.003 & $0.06(0.02,0.11)$ \\
\hline
\end{tabular}

*Effects were estimated for the comparison of live birth rate between women with 39 years old and 32 years old. 


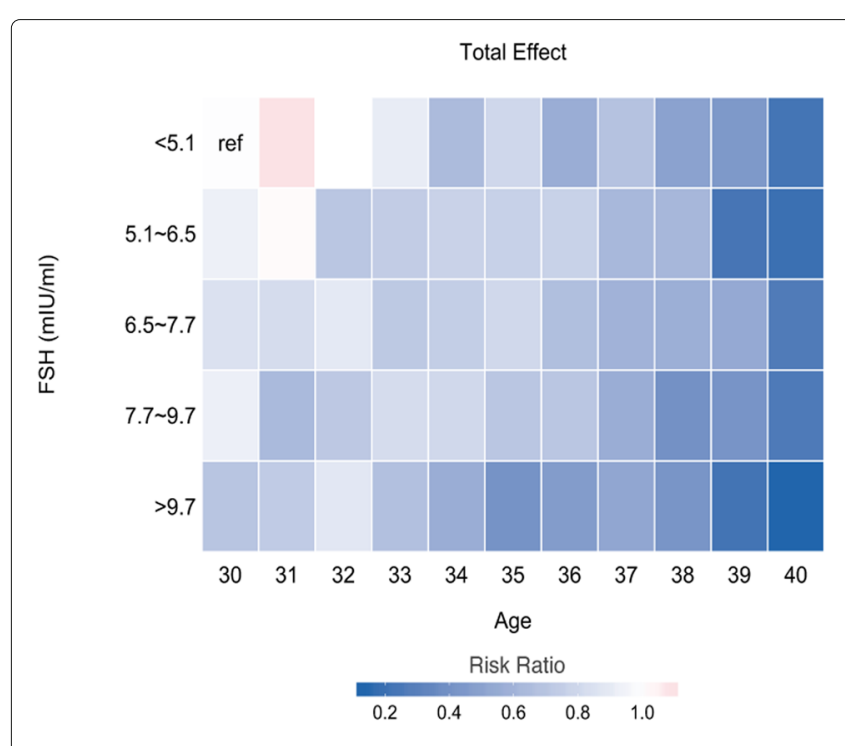

(a)

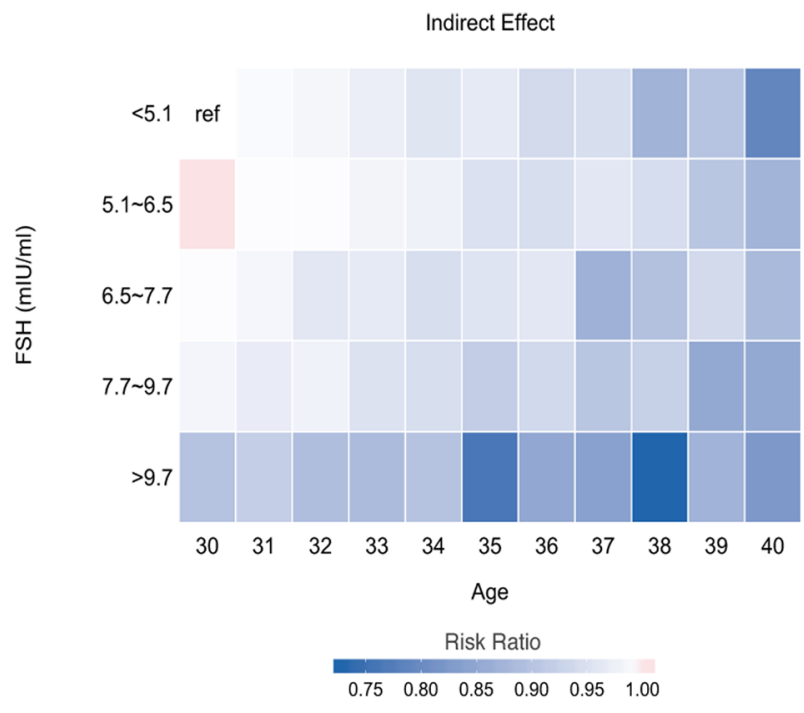

(c)

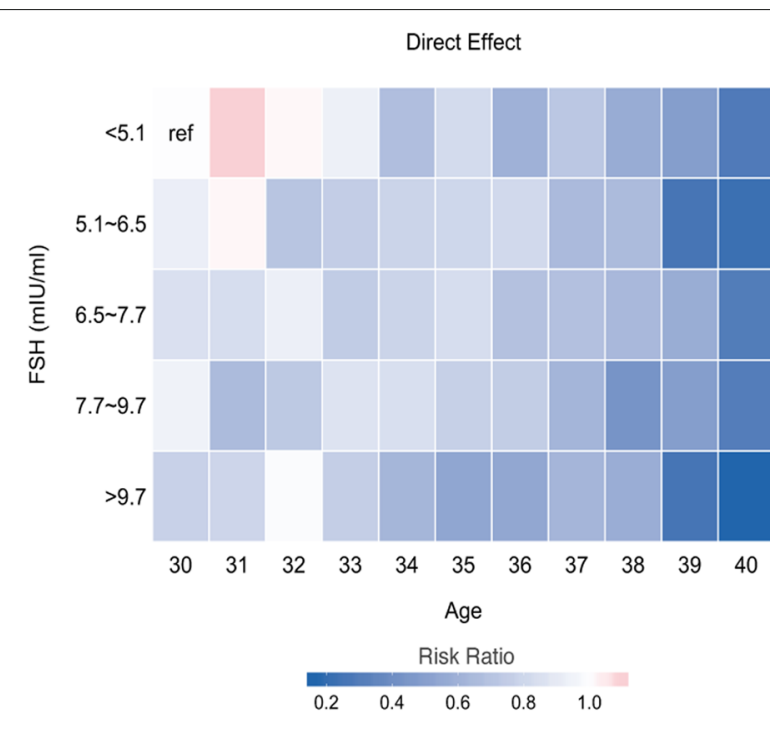

(b)

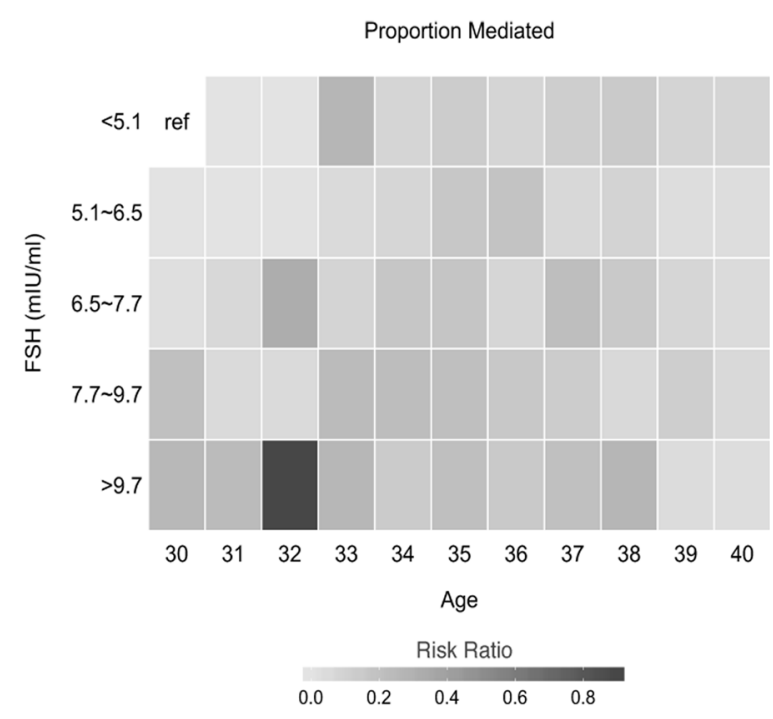

(d)

Fig. 2 Estimated $\mathbf{a}$ total effect, $\mathbf{b}$ direct effect, $\mathbf{c}$ indirect effect and $\mathbf{d}$ proportion mediated with AMH as the mediator for patients from different age groups combined with different FSH groups

While the granulosa cells only develop the FSH receptors during the development of secondary follicles, AMH expression starts in the granulosa cells of early primary follicles, inhibiting the recruitment of primordial follicles $[31,32]$.

Our study has several limitations. Since patients with poor prognosis were more likely to undergo multiple cycles before they achieve live birth, they may contribute a larger proportion of failed cycles in the dataset. This may in return underestimate the mediation effects of AMH (or FSH), and the likelihood of live birth. In addition, although the center-level heterogeneity was adjusted by including the random effects for each center in all models that we applied to characterize the association of live birth and AMH (or FSH), we were still unable to account for the differences in AMH (or FSH) assay due to the diversity of geographic areas encompassed and centers queried.

\section{Conclusion}

In conclusion, the results of this study suggest that the age effect on live birth was mediated more by AMH than by FSH, and that the mediation effect through AMH can 
be further modified by FSH regardless of women's age. More importantly, these results provide a new perspective elucidating the mechanism of IVF successful rate by age, and motivate additional analyses to understand mechanisms that could describe the interplay between age and intermediary factors leading to live birth.

\section{Supplementary information}

Supplementary information accompanies this paper at https://doi.org/10.1186/s12884-020-02875-2.

Additional file 1: Sensitivity analyses, in order to assess the robustness of our estimates.

\section{Abbreviations}

AMH: anti-mullerian hormone; BMI: Body mass index; Cl: Confidence interval; DE: Direct effect; E2: Estradiol; FSH: Follicle Stimulating Hormone; IE: indirect effect; IVF: In vitro fertilization; OR: Odds ratio; RR: Risk ratio; SD: Standard deviation

\section{Acknowledgements}

The authors would like to thank Dr. Yi (Jamie) Zhang for her contributions to this study. The authors also thank PracticeHwy.com Inc. for permission to use their data and are grateful for Jawid Rahimi and Victor Escott for their assistance in sorting and exporting data from elVF.

\section{Authors' contributions}

$\mathrm{HCH}$ carried out the implementation based on the designed model and wrote the manuscript. JYS assisted with sensitivity analysis and helped perform the mediation analyses. SW provided the data and helped supervise the findings of this work. YTH conceived the presented idea and supervised the project. All authors have read and approved the final version of the manuscript.

\section{Funding}

The study was supported by Academia Sinica (06T-1070830-1Q). The funding body played no role in the study design, data collection, data analysis or interpretation of findings.

\section{Availability of data and materials}

The datasets used and/or analysed during the current study are available from the corresponding author on reasonable request.

\section{Ethics approval and consent to participate}

The study was approved by Care New England - Women \& Infants IRB Institution. (Case ID: 948046-1) Since the dataset we obtained was completely de-identified and not patients of Women \& Infants Hospital, it was determined as non-human subject research by IRB committee. No administrative permissions and/or licenses were acquired by our team to access the data used in this research.

\section{Consent for publication}

Not applicable.

\section{Competing interests}

The authors declare that they have no competing interests.

\section{Author details}

${ }^{1}$ Institute of Statistical Science, Academia Sinica, 128 Academia Road, 11529 Taipei, Taiwan. ${ }^{2}$ Women and Infants Hospital in Rhode Island, 101 Dudley St, 02905 Providence, RI, USA.

Received: 27 October 2019 Accepted: 12 March 2020

Published online: 09 April 2020

\section{References}

1. Scott R, Toner J, Muasher S, Oehninger S, Robinson S, Rosenwaks Z. Follicle-stimulating hormone levels on cycle day 3 are predictive of in vitro fertilization outcome. Fertil Steril. 1989;51(4):651-4.
2. Broer SL, Broekmans FJ, Laven JS, Fauser BC. Anti-müllerian hormone: ovarian reserve testing and its potential clinical implications. Hum Reprod Update. 2014;20(5):688-701.

3. La Marca A, Stabile G, Artenisio AC, Volpe A. Serum anti-mullerian hormone throughout the human menstrual cycle. Hum Reprod. 2006;21(12):3103-7.

4. Streuli I, Fraisse T, Chapron C, Bijaoui G, Bischof P, De Ziegler D. Clinical uses of anti-müllerian hormone assays: pitfalls and promises. Fertil Steril. 2009;91(1):226-30.

5. van Rooij IAJ, Broekmans FJM, te Velde ER, Fauser BCJM, Bancsi LFJMM, Jong FHd, Themmen APN. Serum anti-Müllerian hormone levels: a novel measure of ovarian reserve. Hum Reprod. 2002;17(12):3065-71. https:// doi.org/10.1093/humrep/17.12.3065.

6. Broekmans F, Kwee J, Hendriks D, Mol B, Lambalk C. A systematic review of tests predicting ovarian reserve and ivf outcome. Hum Reprod Updat. 2006;12(6):685-718.

7. Blazar AS, Lambert-Messerlian G, Hackett R, Krotz S, Carson SA, Robins JC. Use of in-cycle antimüllerian hormone levels to predict cycle outcome. Am J Obstet Gynecol. 2011;205(3):223-1.

8. MacNaughton J, Banah M, McCloud P, Hee J, Burger H. Age related changes in follicle stimulating hormone, luteinizing hormone, oestradiol and immunoreactive inhibin in women of reproductive age. Clin Endocrinol. 1992;36(4):339-45.

9. Randolph Jr JF, Zheng H, Sowers MR, Crandall C, Crawford S, Gold EB, Vuga M. Change in follicle-stimulating hormone and estradiol across the menopausal transition: effect of age at the final menstrual period. J Clin Endocrinol Metab. 2011;96(3):746-54.

10. Seifer $D B$, Baker VL, Leader B. Age-specific serum anti-müllerian hormone values for 17,120 women presenting to fertility centers within the united states. Fertil Steril. 2011;95(2):747-50.

11. Piette C, De Mouzon J, Bachelot A, Spira A. In-vitro fertilization: influence of women's age on pregnancy rates. Hum Reprod. 1990;5(1):56-9.

12. Hull MG, Fleming CF, Hughes AO, McDermott A. The age-related decline in female fecundity: a quantitative controlled study of implanting capacity and survival of individual embryos after in vitro fertilization. Ferti Steril. 1996;65(4):783-90.

13. Kupesic S, Kurjak A, Bjelos D, Vujisic S. Three-dimensional ultrasonographic ovarian measurements and in vitro fertilization outcome are related to age. Fertil Steril. 2003;79(1):190-7.

14. Baron RM, Kenny DA. The moderator-mediator variable distinction in social psychological research: Conceptual, strategic, and statistical considerations. J Pers Soc Psychol. 1986;51(6):1173.

15. Mackinnon D. Introduction to Statistical Mediation Analysis: Routledge; 2012. https://doi.org/10.4324/9780203809556.

16. Robins JM, Greenland S. Identifiability and exchangeability for direct and indirect effects. Epidemiology. 1992143-55.

17. Pearl J. Direct and indirect effects. In: Proceedings of the Seventeenth Conference on Uncertainty in Artificial Intelligence. San Francisco: Morgan Kaufmann Publishers Inc.; 2001. p. 411-20.

18. Rubin DB. Bayesian inference for causal effects: The role of randomization Ann Stat. 1978;6(1):34-58. https://doi.org/10.1214/aos/1176344064.

19. Imai K, Keele L, Yamamoto T, et al. Identification, inference and sensitivity analysis for causal mediation effects. Stat Sci. 2010;25(1):51-71.

20. VanderWeele TJ, Vansteelandt S. Conceptual issues concerning mediation, interventions and composition. Stat Interf. 2009;2(4):457-68.

21. Lange T, Hansen JV. Direct and indirect effects in a survival context. Epidemiology. 2011;22(4):575-81. https://doi.org/10.1097/ede. ob013e31821c680c.

22. Valeri L, VanderWeele TJ. Mediation analysis allowing for exposure-mediator interactions and causal interpretation: theoretical assumptions and implementation with sas and spss macros. Psychol Methods. 2013;18(2):137.

23. Shih $\mathrm{S}$, Huang $\mathrm{Y}-\mathrm{T}$, Yang $\mathrm{H}-\mathrm{I}$. A multiple mediator analysis approach to quantify the effects of the adh $1 \mathrm{~b}$ and aldh2 genes on hepatocellular carcinoma risk. Genet Epidemiol. 2018;42(4):394-404.

24. Wang S, Zhang Y, Mensah V, Huber WJ, Huang Y-T, Alvero R. Discordant anti-müllerian hormone (amh) and follicle stimulating hormone (fsh) among women undergoing in vitro fertilization (ivf): which one is the better predictor for live birth?. J Ovarian Res. 2018;11(1):60.

25. Nelson SM, Telfer EE, Anderson RA. The ageing ovary and uterus: new biological insights. Hum Reprod Updat. 2012;19(1):67-83. 
26. Fretts RC, Schmittdiel J, McLean FH, Usher RH, Goldman MB. Increased maternal age and the risk of fetal death. N Engl J Med. 1995;333(15): 953-957.

27. Nelson S. M. Biomarkers of ovarian response: current and future applications. Fertil Steril. 2013;99(4):963-9.

28. Group ECW. Fertility and ageing. Hum Reprod Update. 2005;11(3):261-76.

29. Zhu Q, Chen $Q$, Wang L, Lu X, Lyu Q, Wang Y, Kuang Y. Live birth rates in the first complete IVF cycle among 20687 women using a freeze-all strategy. Hum Reprod. 2018;33(5):924-9. https://doi.org/10.1093/ humrep/dey044.

30. McLernon DJ, Steyerberg EW, te Velde ER, Lee AJ, Bhattacharya S. Predicting the chances of a live birth after one or more complete cycles of in vitro fertilisation: population based study of linked cycle data from 113873 women. Bmj. 2016;355:5735.

31. Durlinger AA, Visser JJ, Themmen AA. Regulation of ovarian function: the role of anti-mullerian hormone. Reproduction. 2002;124(5):601-9. https:// doi.org/10.1530/reprod/124.5.601.

32. Visser JA, de Jong FH, Laven JS, Themmen AP. Anti-mullerian hormone: a new marker for ovarian function. Reproduction. 2006;131(1):1-9.

\section{Publisher's Note}

Springer Nature remains neutral with regard to jurisdictional claims in published maps and institutional affiliations.

- fast, convenient online submission

- thorough peer review by experienced researchers in your field

- rapid publication on acceptance

- support for research data, including large and complex data types

- gold Open Access which fosters wider collaboration and increased citations

- maximum visibility for your research: over $100 \mathrm{M}$ website views per year

At $B M C$, research is always in progress.

Learn more biomedcentral.com/submission 